\title{
Epistemic Cultures: \\ Forms of Reason in Science
}

\section{Karin Knorr Cetina}

Inside the Epistemic: Rationality and Context

Rationality is a conspicuous yet neglected phenomenon. It has received much attention from philosophers and some social scientists, who have treated rationality as the hallmark of science, of economic action, or of modernity in general. Yet with all this attention, rationality has been conspicuously taken for granted, and its taken-for-grantedness has licensed a neglect of the practical activity of rational action. In other words, rationality has been a resource rather than a topic of analysis. To be sure, some fields have long been preoccupied with specifying the rules of rationality which are capable of unifying - or reconstructing human endeavors within a domain of activity; but they have done so at the cost of neglecting to seek to understand the actual practice of these activities. For example, philosophers of science have relegated the vast territories of the "real" behavior of scientists to a marginal existence, in exchange for the doubtful benefits of the rational reconstruction of scientific theory choice. Economic theorists appear perennially involved in searching for "indisputable" definitions of rational choice which can serve as algorithmic devices in economic modeling, but they leave unexamined essential empirical aspects of real business-firm and market operations. ${ }^{1}$ In a paraphrase of Hegel, Bruno Ingrao has recently described the content of economic theory as a "representation of rationality as it is in its eternal essence, before the creation of nature and of a finite mind" $(1989,120)$.

As the many controversies over the content and definition of scientific and economic reason suggest, rationality is an interesting topic of investigation. But must this investigation be limited, as often in economic theory, to the mental and computational specification of rational calculation and constrained optimization as required by formal models to

Correspondence may be addressed to the author, Faculty of Sociology, University of Bielefeld, P.O. Box 8640, D-4800 Bielefeld 1, Germany.

1. For a review of methodological traditions in economics see Weintraub 1985 and de Marchi 1988. For an overview of realism and reference-or the lack thereof-in economic theory see Mäki 1989. 
test properties of a model's structure? Or, as with philosophers of science, to the critical deployment of principles of choice and procedure, derived from thought experiments, to idealized versions of scientific theory?

Recently a philosopher of science summarized his field by saying: "Philosophers' theories of science are generally theories of scientific rationality. The scientist of philosophical theory is an ideal type, the ideally rational scientist. The actions of real scientists, when they are considered at all, are measured and evaluated by how well they fulfill the ideal. The context of science, whether personal, social or more broadly cultural, is typically regarded as irrelevant to a proper philosophical understanding of science" (Giere 1988,3). This comment is interesting not only because it shows the extent to which philosophy holds science to be synonymous with rationality but also because it shows the full force with which rationality is seen to be synonymous with untextured, contextless procedure. In the philosophy of science rationality is characteristically set up against "context" in a center-periphery arrangement, with rationality perceived to be at the core of the conduct of interest and "context" relegated to the fringe." Context," of course, is a catchword for all that is not strictly rational, which, as the quote specifies, includes the "personal," "social," or "more broadly cultural."

Strangely enough, many of the fields which study these "personal," "social," or "cultural" phenomena have cooperated with this definition. The sociology of science, for example, has long defined its goal in terms of the analysis of the external "scenery" within which what is really "science" takes place. Merton, who is widely recognized as the father of the sociology of science, proposed that we only investigate the "mores" of science, leaving its methods and contents to philosophy for rational investigation. ${ }^{2}$ The sociology of knowledge, too, has traditionally framed its goal in a way which externalizes context. To be sure, the sociology of knowledge has always included the content of the knowledge it investigates in its analyses. But it nonetheless has construed society as an external influence upon knowledge. The sociology of knowledge has effectively asked the question how society-context-gets into knowledge. For example, Mannheim (1936) asked how the social position of an individual (or a class) determines what that individual (or class) thinks. And recent proponents of the sociology of knowledge have raised a similar question by studying how social interests and class interests sustain knowledge claims (they answer the question differently, of course; see Barnes 1974, Bloor 1976).

2. For an exposition of Merton's program for the sociology of science see Mulkay 1979, 21ff. See also Merton 1973.
The view that "context," "society," or "culture" is at the fringe of science/knowledge and that the question is at best how these domains "affect" scientifically rational procedure is strangely self-restrictive in that it ignores how "context" is always part of science-not as an external determinant of something that is pure scientific method or pure thought, but as part of the internal organization and performance of knowledge-developing and knowledge-grounding procedures.

I want to propose that we see scientific method as a heavily textured phenomenon rather than as the mere execution of some philosophically intuited standard of reason. Context or, in a broad sense, "culture" is inside the epistemic, and the sociology of knowledge, or perhaps we should rather say the study of knowledge, must also concern itself with the cultural structure of scientific methodology. To be sure, some recent sociologists of science have already made a start-for example, Harry Collins in his work on replication (1985), and various laboratory studies as well as recent studies of experimentation (Latour and Woolgar 1979; Knorr Cetina 1981; Lynch 1985; Godding et al. 1989). Yet we are still lacking a coherent analysis of the "truth-finding" machinery of the natural sciences, of what its features are (in the old terminology, these would be "hypothesis-testing," "proving" "argumentation," and the like), of how these features hang together, and of how they connect, if they do, to what has always interested us, namely "social relations."

To pursue such a goal one must move inside the epistemic space within which scientists work and identify the tools and devices which they use in their "truth"-finding navigation. Not surprisingly, ${ }^{3}$ what we find there is not the clear and austere progress of verifications (or falsifications, or explanatory capacities) we might expect, but the "untidy" goings-on of various businesses of experimentation. When added together, these goings-on in a particular domain form what one might call an epistemic culture. The notion foregrounds not only a "relatedness" and "clustering" of various parts, but also a "disunity" of science in regard to epistemic practice. Such a disunity is rarely accepted in philosophy of science ${ }^{4}$ and has not been demonstrated in sociological studies of the area. Differences in gradation in regard to the "advancement" or "maturity" of a discipline notwithstanding, virtually nobody appears to doubt the unity of science with respect to the relationship which obtains between scientific "findings" and objects or events in the real world. Whether this relationship has been conceived of in realist, logical empiricist, pragmatist, or skepticist terms, it has always been thought of, it seems, as a "shared-by-all" connection. But is it really

3. After several years of "close-up" studies of scientific work, this is no longer surprising, though it may have been at an earlier time.

4. For an exception see Hacking 1983. 
shared by all? And is the unitary narrative about "the scientific method" sustainable once one follows the directive proposed above and moves inside the epistemic space?

In what follows I want to illustrate the cultural structure of scientific methodology and the disunity it implies by drawing upon two laboratory studies which we conducted in recent years, one in experimental particle physics and the other in molecular biology. 5 Here I must restrict myself to some aspects of what might loosely be called the organization of openness and closure-openness being understood as referring to the resistances and obstacles scientists encounter in their work, and closure to their dealing with these difficulties. More concretely, I focus on different forms of reason and rationality in the two sciences and their relation to obstacles in the form of circularities and contingencies. In concluding, I add some speculations as to how local differences in epistemic practice anchor differences in these sciences' relations with the real world. But the issue of course is not to propose an alternative to existing conceptions of this relationship. It is rather to recover "epistemology" from studying finished products (restrictively defined as scientific theories) and criteria for choosing between these products which further scientific progress. The point is to concern epistemology with process-with the concrete, mundane, everyday practices of inquiring and concluding through which participants establish, for themselves and for others, knowledge claims.

\section{Where Reason Dwells: The Problems of Circularity and Contingency}

The first thing to note about notions of reason and rational procedure is that these appear to thrive in a particular kind of environment, notably in situations of dilemma or, to put it more strongly, in situations of paradox. We found considerations of rationality linked to the following two situations:

1. Situations marked by the existence of a circular relationship between an outcome and the method of inquiry, such that to obtain a desired outcome one needs to optimize the method of achieving a result, but to optimize the method one needs to know the outcome of the work in advance.

2. Situations marked by the existence of an internal relationship of contingency between two desired research utilities, such that the pursuit or attempted optimization of one utility prevents one from pursuing or optimizing the other. 5. These studies are in the process of being written up and published (Knorr Cetina et
al. 1991, Amann 1990). For earlier results see Amann and Knorr Cetina 1988, 1989; Knorr al. 1991, Amann 1990). For earlier results see Amann and Kno
Cetina et al. 1988; and Knorr Cetina and Amann 1988, 1990.
Both situations occur in both the desciplines we studied, though negatively contingent outcomes appear to be more prominent-and are more often articulated-in particle physics. And both forms of dilemmas present formidable difficulties for any kind of simple, procedural rationality in science. Let me first illustrate the circularity problem by an example from molecular genetics. ${ }^{6}$

Gel electrophoresis is a method for separating DNA and RNA fragments of different length in a gel on which an X-ray film is exposed. As a result of the procedure one gets blackish and whitish bands which are most clearly distinguishable in the middle of the matrix which the film represents (for an example see figure 1). At the bottom of the film the bands tend to be drawn apart, and on top they tend to stick together and may become indistinguishable. Thus, to obtain a good resolution and highly analyzable and publishable results, the bands of interest should be placed in the middle of the matrix. And to achieve this, the gel run must be stopped (the voltage under which a gel is run must be turned off) exactly when the fragments of interest appear in the right place. This, however, is possible only if one knows the length of the expected fragments - that is, of the bands - in advance. But this of course is never the case, as it is precisely the goal of the gel run to determine the length of the fragments one is interested in. Thus the circular relationship between gel run and its outcome results from the fact that the optics of the experiment (of the gel) can only be optimized through knowledge of the expected bands, while at the same time this optics is already presupposed in any attempt to determine these bands.

Analogous circular self-referential relationships occur in particle physics. The example I now offer is also, at the same time, an example of a relationship of contingency between two research goals or utilities. In the experiment we observed, protons and antiprotons are accelerated in a pp collider and hurled against each other, thereafter decaying into secondary and tertiary particles which travel through different detector materials before they get "stuck" in the outer shell of a calorimeter. Detectors can "see" the traces left by these particles and announce their presence to a "read-out chain." But this can take place only after a "trigger" has determined which of the particles that came by were "interesting" from the point of view of the experiment, that is, which events fulfilled certain energy-threshold and other requirements; uninteresting events are ignored. However, trigger calculations in regard to which event is "interesting" (has sufficient energy) need time, during which the read-out chain is turned off and the detector does not "look at" the incoming events. This is the "dead time" of the experiment,

6. For a full ethnography of the molecular biology laboratory from which this example is derived see Amann 1990. 


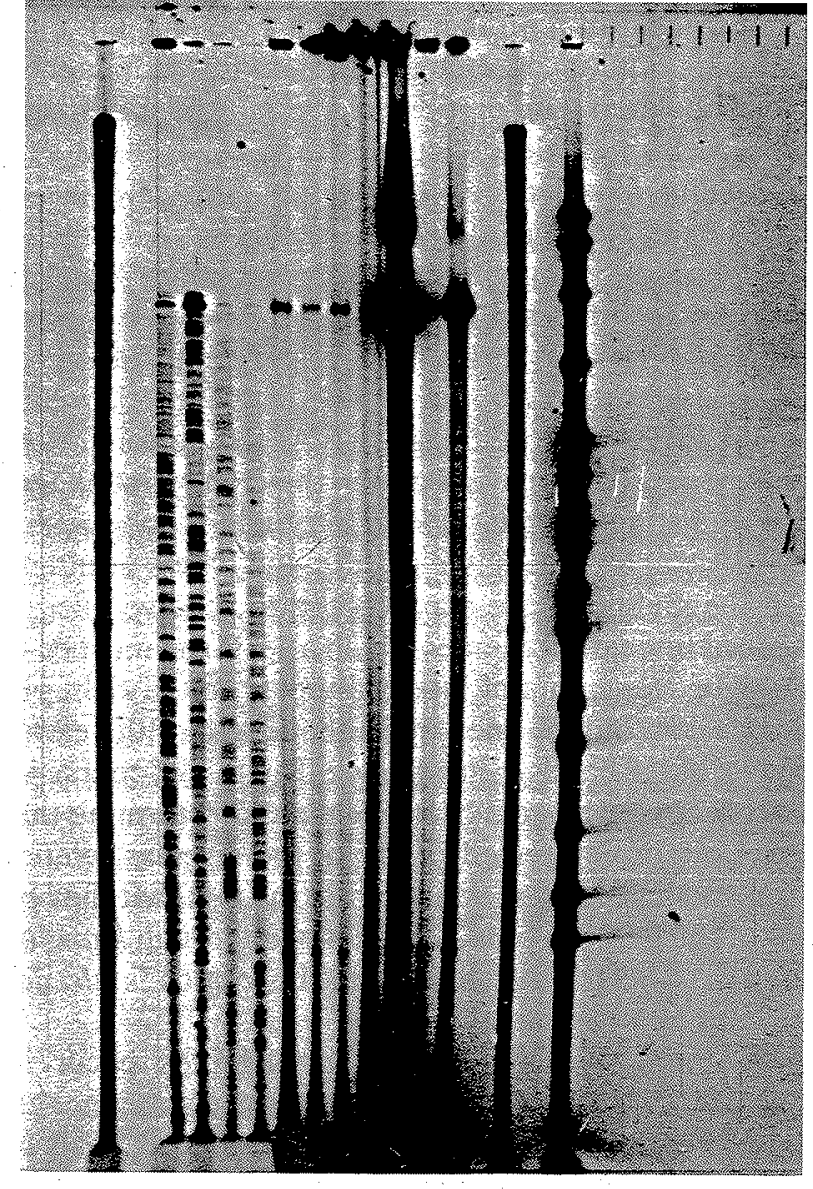

Figure 1. Example of an autoradiograph film from the molecular biology laboratory

which is increased by the time participants need to change data tapes, by the event length, and by other occurrences. The higher the dead time of the experiment, the smaller the rate of interesting events written to tape, and the smaller the chance to find the "top quark," which is the purpose of the experiment. Threshold decreases can increase the rate of accepted events and decrease the dead time. But they also increase the number of background events written to tape, and one quickly ends up flooding the read-out system and the computer with a rate which cannot be handled either by off-line operations or by the physicists who analyze the material. Thus the rate and the dead time (the background and the signal) cannot both be optimized at the same time-which is the con- tingency I referred to. And there is also a problem of circularity equivalent to the one in the preceding example, in that if the mass of the top quark and the background to the top were known, then the rate and the dead time could be fine-tuned to the top's requirements. But of course they are not known; to determine the mass of the top quark is the goal of the experiment.

The circularities and contingencies illustrated in these examples are basic epistemic problems without direct solution; for example, in the case of contingent research utilities no Pareto improvement is possible. Besides, these are problems which occur routinely in numerous variants and which, for that reason, complicate scientific inquiry on an everyday level. Notions of reasonable procedure in scientific activities must be seen in the light of these difficulties. They appear to respond to them, and they do so in different fashions. But what are these notions? And how do they differ between the two fields investigated?

\section{"Mirroring" the Phenomenon and Trade-off Operations}

Consider first particle physics. Two strategies are apparent in this field: a framing strategy and an exchange strategy (calculative strategy).

The framing strategy. The framing strategy refers to the strategy of particle physics to cure reflexivity and similar problems through considering comparable data and objects which serve as a frame of reference in dealing with the problem. For example, contingent results from one detector will be framed routinely by the results of a second detector, and data obtained from one data run will be put into perspective by the results from another, special run. Measurement results are frequently published by including, in graphic representations, the distributions published by other experiments (for an example see figure 2).

Physics theory and Monte Carlo simulations furnish further reference measures in the battle against contingencies. In the end whole experiments frame each other (see figure 3). Particle physics has the habit of setting up parallel experiments which are allowed to compete against each other in the same laboratory: for example, the experiments UA1 and UA2, and the four LEP experiments at the European Center for Particle Physics (CERN) in Geneva. On an international level, races (to find the $\mathrm{W}$ and $\mathrm{Z}^{0}$ intermediate vector bosons, to find the top quark) are regularly staged between the three or four top laboratories in the world. A recent example is the race between the experiments UA2 at CERN and CFI at FERMILAB, Chicago (which has been documented by the BBC). ${ }^{7}$ Particle physics seems to deal with the closedness of its system

7. Videotapes of the film, which the BBC broadcast in 1989-90, are available from the CERN Public Relations Department. 


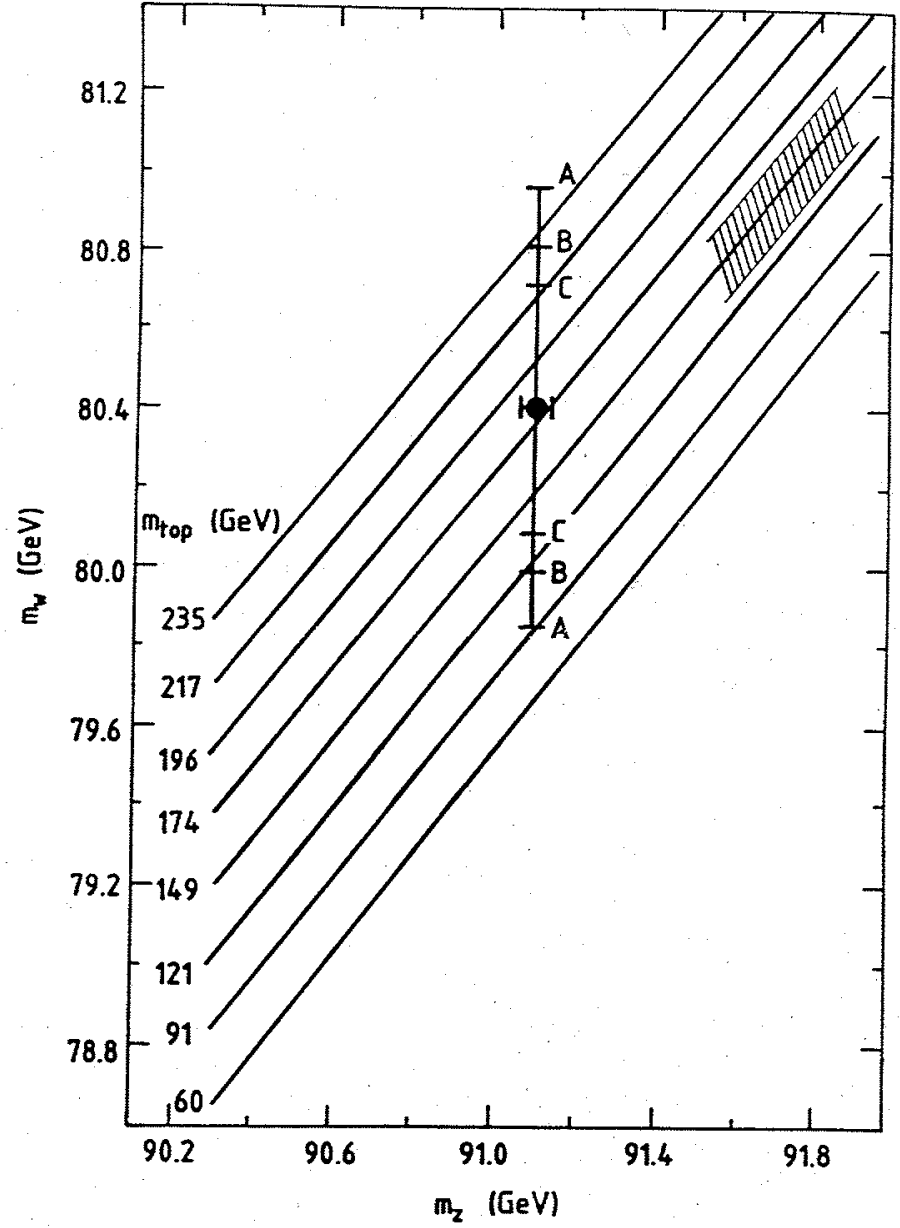

Notes: $m_{z}$, as measured at LEP, vs. $m_{w}$, as measured by $U A 2$ using the $m_{w} / m_{z}$ ratio and the LEP value for $m$

A) present data; B) two-fold increase of the event samples; C) four-fold increase of the event samples. The lines of constant $m_{\text {top }}$ were calculated using a Higgs mass of $100 \mathrm{GeV}$. The shaded area represents theoretical uncertainties associated with a variation of the Higgs mass between 10 and $100 \mathrm{GeV}$.

Figure 2. Results from one experiment (LEP) framed by those of another (UA2) for different event samples $(A, B, C)$.

(see below), which manifests itself in contingencies and circularities, by continously inventing mirroring strategies.

The exchange strategy. If, in the first case, rationality is a question of the multifaceted mirroring of experimental components, in the second case it is a process of utility trading and transformation. Recall that I

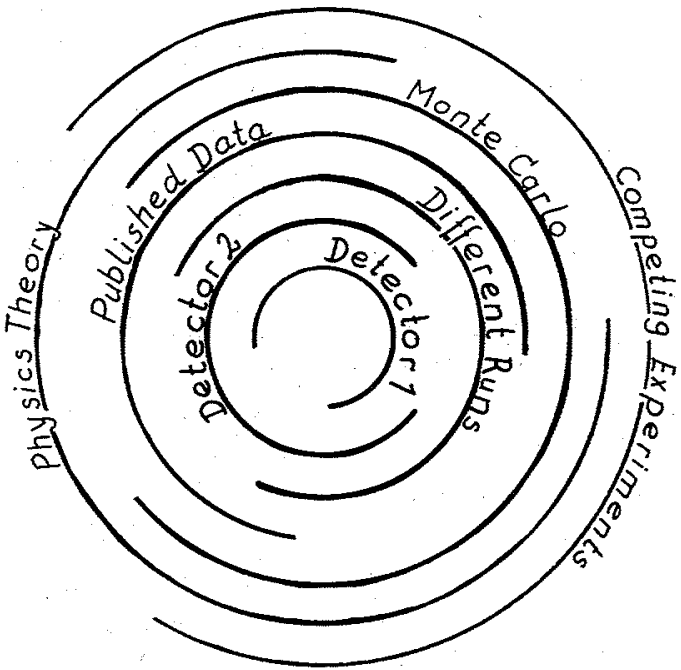

Figure 3. Framing strategies in particle physics. The results of one detector or one data-taking run are framed by those of others, results from one experiment are framed by the published results of others, experimental measurements are framed by Monte Carlo simulations, and whole experiments which are currently operational are framed by other such experiments.

have defined contingency in terms of a negative relationship of dependence between two desired goals, or research utilities, such that one utility can only be obtained or optimized at the cost of the other. In this situation particle physicists resort to a strategy of commerce and exchange: they balance research benefits against each other, and they "sell off" those which they think that, on balance, they may not be able to afford. Particle physicists refer to this commerce with research benefits as "trade-offs." In the experiment we observed, they traded off tracking particles against electron identification; speed in finding tracks against precision in track identification; time needed for calibration against granularity of the detector; performance of the calorimeter against cost; dead time against background reduction; and so on.

A detector is a layered deposit of these sales, a balance of trade of various exchanges turned into material form. It is a checkered assemblage of numerous blemishes which are nothing else than the price tags these trade-offs have carried. Now it is a cognitive economy through which the trading proceeds, a commerce realized through a traffic of reasons which binds together members of an experimental collaboration. Meetings are the occasions in which many of these deals are closed, in which trade-offs are presented, reasoned out, and collectively endorsed. The exchanges are kept track of through bookkeeping, that 
is, through working out algorithms which quantify the payoffs and losses of any given exchange in terms of calibration constants, efficiency measurements, measurements of gain, and so on. The commerce has a social structure in that some people are strategically placed to observe the whole trade-off operation. Interestingly, these people are not necessarily physicists but may instead be computer wizards; in the experiment UA2 at CERN it is the head of off-line operations who prepares the final data summary tapes which every member of the experiment uses in the end. He assembles and banks the measurements and algorithms which quantify the experiments' exchanges.

Now reasons are the currency but Reason (with a capital R) is also the upshot of these continuous trade-off calculations. The construction that participants place upon the situation is that of the proverbial Reasonable Man. In calculating and trading, the participants try to effectuate what the Reasonable Man would do. The point here is that the Reasonable Man is above all a merchant and a compromiser. He or she is a skilled trader rather than someone who-in the name of science-is shy to buy and sell. He or she does not trade indiscriminately, but his/her discrimination is not, as a rule, along the lines of "scientific" versus "unscientific" considerations. In fact "scientificity," epistemic reasons, are constantly sold off for money, time, competitiveness, manageability, and other "nonscientific" - yet necessary - goods. One of the criteria for triggering and in general for background cutting is to reduce the data one is handling to a "manageable" size-which may be the size that allows the data to be processed on the computer overnight so that they can be analyzed by a physicist in the morning (here particle physicists practice a kind of "Merleau-Ponty-ism"). ${ }^{8}$ It appears that experiments in particle physics may gain power through their various utility transformations, through this form of "open" rationality. Like framings, trade-offs are strategies for producing truth.

\section{The Phoenician and the Baconian Forms of Reason}

If we now turn to molecular biology, the picture changes dramatically. One difference is that in particle physics circularities and contingencies appear to be more massively present (bookkeeping enhances this impression), more elaborately answered, and more frequently commented upon. This sort of "elaborateness" of these phenomena is lacking in molecular genetics. What is also lacking is the differentiation of two

8. Merleau-Ponty's phenomenology of perception includes a theory of the body as a frame of reference for human experience and human endeavors. In relating data reduction
to their physical capabilities and needs, particle physicists provide an illustration-albeit somewhat vulgar-of Merleau-Ponty's claims. See Merleau-Ponty 1962. different problem-handling strategies, that is, of a framing strategy and a strategy of utility exchange and transformation.

Molecular biologists attack the problem of circularity by leaving it to the individual scientist to develop a "sense" for a reasonable strategy in response to the challenge. Thus the problem is turned into a private one-one for which the individual scientist is responsible and can be held accountable - and this "privatization" is sustained by the collectively shared assumption that "systematic" strategies are too time-consuming and therefore not conducive to reaching molecular biologists' goals. In the example illustrated in figure 1, a systematic (methodical) strategy would be one that would require scientists to optimize the optics of the gel run by running several gels simultaneously and switching them off each at a different time, in order to identify the run time best suited to placing the resulting bands in the middle of the matrix. This procedure, however, means that the number of reaction mixes which have to be prepared has to be multiplied by the number of parallel gel runs; and for each reaction mix; there has to be a purified protein extract; and so on. It is not only that the number of tasks to be performed quickly grows with this procedure; it is also that each step is affected by the same difficulty and uncertainty of having to be made to work in the absence of the outcome which provides the clue as to how the procedure is to be made to work.

Molecular biologists situate themselves in their strategies (see figure 4) somewhere between what they consider a "chaotic" and a "methodical-systematic" procedure such as just described. Both strategies are regarded as irrational, if for different reasons. The intermediate rationality which molecular biology prefers draws heavily upon an individual's experience: individuals are expected to synthesize from their own earlier experience the prognostic knowledge they need to repair circularities and contingencies. In this connection one can point to the narrative culture of the lab-to the "traffic" of stories which encapsulate individual experience and through which this experience circulates in the lab.

The required "sense" of successful procedure, of this embodied experimental knowledge, remains implicit. It is partly encapsulated within the person and partly encapsulated within the narrative culture of the laboratory. I would like to call this form of reason "biographical": it is sustained by a scientist's biography, the archive and store of his or her professional experience. In contrast, particle physicists' forms of reason include more awareness of the need for trade-offs and more openness to utility considerations which transcend science-these are also public reasons in the sense of being both explicit and literate. The hallmark of the trades particle physists engage in is not only that ex- 


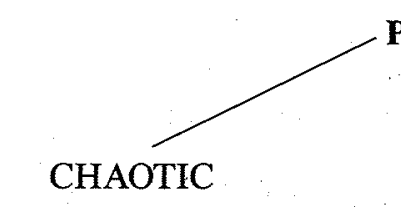

Proced

For example:

- Too many tasks pursued simultaneously

- Lack of coordination

- No documentation

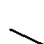

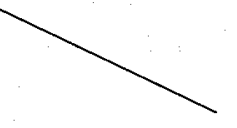 \\ SYSTEMATIC}

(breaks up circularity)

Risk management:

- Dividing whole into component tasks

- Stepwise optimization of components

\section{BIOGRAPHIC RATIONALITY}

(circularity-embracing)

Risk management:

- Person as experience synthesizer

- Person as narrator

Figure 4. "Chaotic," "systematic" and (biographically) "rational" procedures in molecular biology. With the "systematic" strategy, circularities and contingencies are systematically broken up and substeps are optimized; with the "rational" strategy problems of circularity and contingency are approached holistically and by requiring the scientists to synthesize the knowledge they need to repair circularities from their own earlier experience.

changes are effectuated, but also that the payoffs and losses of these exchanges are computed, recorded, and fed back into the analysis. Similarly, the framing strategy (which is frequently tied to trade-off considerations) turns around the possibility of comparing between frames, of computing their differences, of calculating from a reference distribution what a measurement should be. If one wanted to draw an analogy between these forms of reason and Bernstein's "restricted" and "elaborated" codes, ${ }^{9}$ one would have to say that the physicists'. Phoenician

9. For an exposition of Bernstein's model of language learning see Bernstein 1965. Bernstein distinguishes between two basic categories of speech in linguistic and sociological terms. The first, called "restricted code," arises in small-scale local environments in which speakers are tied together by common experiences and assumptions and hence need not (and cannot) fully explicate their meanings. The second categry ans and hence need not (and cannot) fully explicate their meanings. The second category, called "elaborestricted code has been associated with lower-class languasumptions explicit. The elaborated code has been tied to the more "schooled" and educated classes. form of reason is elaborated, whereas molecular geneticists' Baconian version is restricted and indexical as a result of being embodied within the person and the oral culture of the group.

\section{Internal Reconstruction (Self-Reference) and Adaptation}

In drawing these analogies one invites speculation as to which form of reason is more circumspect and "progress-prone." However, rather than pursue these questions I want, in concluding, to draw attention to some other details and their consequences in regard to the "disunity" of science mentioned at the beginning of this essay. I begin again with particle physics. The point is that the physicists' literary form of reason goes hand in hand with a loss of the empirical whereas molecular geneticists' biographic form of reason does not. First, natural objects (cosmic particles) or quasi-natural objects (debris of smashed particles) are admitted to an experiment in particle physics only rarely, perhaps for a period of months in an experiment that may take anything between eight and sixteen years. Second, these objects are, for the physicist, "unreal" in the sense that they are not visible and can only be established indirectly, through processing the "footprints" or traces (electric charges, "holes" from knocked-out electrons, etc.) that particles leave when they fly through different pieces of equipment (detectors). In a rather literal sense, experimental particle physics is a science that deals with signs, or as the physicists say, signatures of events rather than with the events or objects themselves. Third, there is the added complication, with some machines especially but with all to some degree, that the traces of particles one is interested in are muffled by the traces of "uninteresting" particles, the "background." Since the signal-to-background ratio is very low, it is as if billions of stampeding animals had left footprints on a trail from which those of only a handful of animals need to be extracted (in CERN's experiment UA2, fewer than ten top candidates were expected out of 6 billion original events). Fourth, the traces that events or objects leave, their measurements, are seen to be heavily dependent upon the many contingencies and blemishes of the measurement machinery (e.g., the detectors and electronics) and hence cannot be taken seriously as they stand. Rather they must be recalculated in the light of reference measurements and reference considerations. These include measures of detector deficiencies and idiosyncrasies, of "aging" and deterioration of equipment, reference measurements produced by contrasting or supplementary equipment or data runs, and "monte carloed" theoretical expectations. The "framing" mentioned above in regard to circularity and contingency is part of these calculations. "Signals"-i.e., particle physicists' data—are obtained only after the signs 
left by particles and particle debris have been heavily processed through "production" and "reconstruction" programs which perform these recalculations. As a consequence of this whole situation, the "empirical" in experimental particle physics appears to be firmly embedded in a network of anticipation, simulation, and recalculation-to a degree at which it seems no longer the phenomenon itself which is at issue but only its reflection in the light of the internal megamachinery which envelops and tracks down physical occurrences.

There is an analogy which I think is appropriate to the "truth-grounding" strategy of experimental particle physics: that of the brain as an informationally closed system. The neurophysiology of cognition which has been developed by Maturana, Varela, and others is based on results developed in the nineteenth century, according to which states of arousal in a nerve cell represent only the intensity, but not the nature of the source of arousal. ${ }^{10}$ Maturana and Varela applied these results to the study of perception and concluded that "living systems" are cognitive systems which are energetically open but informationally closed. Perception, for example, is accomplished by the brain, not the eye, and the brain can only construe reality from signals of light intensity by reference to its own previous knowledge and by utilizing its own electrochemical reactions. To phrase it differently: in perception the brain interacts with its own internal states and not with those of an external environment; it reconstructs the external world in terms of these states-and in order to do so it "observes" itself. Consciousness is a function of a nervous system that is capable of recursive self-observation.

Like the brain, experimental particle physics appears to be uniquely absorbed with its own complicated circuitry and its previous and parallel internal states. The traces left by particles correspond, literally; to differences in energy or current from which particle physics reconstructs, through reacting with itself, information in the form of raw data (not physics results!). In order to be able to perform these reconstructions particle physics spends most of the time of an experiment learning about and "understanding" itself-by which I mean observing, improving, controlling, and testing the devices (mechanical and electronic instruments, software, mathematical calculations, etc.) which it fabricates. The grounding of empirical findings is established through selfreference, self-contexturing, self-organization-through establishing, maintaining, observing, and constantly reincorporating knowledge of itself. It is a grounding that rests upon recursiveness and self-explanation.

10. For an exposition of the theory see, for example, Maturana and Varela 1980.
If we now turn to molecular genetics we find nothing which corresponds to the loss of the empirical and to the recursive epistemics of particle physics. Quite the contrary: molecular genetics appears to base progress upon maximizing contact with the empirical world. First, (quasi-) natural objects are sought out and encountered on a day-to-day basis. They have to be cultivated and bred, they must be cared for, kept warm, nourished, and watched. Much of the success of a laboratory in molecular genetics depends on how well it manages to nurse and attend to the many ingredients of experiments. In many respects a molecular genetics laboratory is a store of these ingredients, and participants are their long-term caretakers. Second, these objects are constantly manipulated in experiments. In fact, in molecular biology single "experiments" consist of tens and often hundreds of separately attended-to substeps and subsubsteps, all involving separate materials and a distinctive, identifiable procedure. Although no attention is drawn to these substeps in publications (except in special cases), from the point of view of the performer they constitute the bulk of the work. Third, the progression of the work in these subordinate phases and its outcomes are noticed by skilled researchers, even though the processes may not be consciously observed. The features and circumstances of performance register with participants and can be recalled from subconscious memories should the need arise. This feature enables participants to "backtrack" a sequence of events in the many cases where the outcome of a series of substeps does not "make sense."

What it all adds up to, I think, is that scientists and "things" in the molecular genetics laboratory are joined together in interactional/behavioral circuits, in systems of conjoint reaction and reciprocity in which neither behaves independently of or completely dominates the other. These systems are held together by a sensory reciprocity, and they are based on "sensibilities" rather than conscious reflection. The scientist's behavior, of course, is not cognized by the specimen he or she deals with; but nor are these scientists' responses best thought of in terms of mental labor. The mind tends to be involved, of course, but more in the sense emphasized by Ryle as an embodied, practical skill (Ryle 1971). In fact, as implied above, the mind is often drawn upon as a kind of memory organ, a tablet on which the behavior of a specimen inscribes itself without participants' conscious effort or awareness. What one might think of here is G. H. Mead's notion of a "conversation of gestures," which he used to illustrate the behavioral reciprocity of action and reaction established between two persons boxing, and which happens without conscious premeditation (Mead 1967).

Thus molecular geneticists do interact with "the world"-as it is featured in the laboratory of course, but this featuring does not preclude 
but rather enhances resistence. They constitute part of a behavioral system in which "things" are not passive receivers but active reactants. As scientists perform on the level of organization of materials, a system is established which affords constant opportunities for mutual reconfiguring, adjustment, and adaptation. What I want to suggest is that within this system there exists ample opportunity for the science to "reform" and reshape itself continuously around the objects it encounters, whether these objects are themselves cultural objects or not. Am I thereby embracing scientific realism? I think not. "Success" in the sense of recoverable, "working" phenomena appears perfectly imaginable as, at least in part, a result of the interactional dynamics I proposed without need to bring into the picture correct theoretical representations. Furthermore, much of laboratory science in molecular genetics neither directly draws upon, nor does it seem terribly involved with establishing, theoretical representations. In molecular genetics, theoretical statements may indeed be post hoc "rationalizations" of materials, with the progress of research being sustained by an infrastructure of interactionally worked out, "world"-accommodating procedures.

The interactional infra-epistemics within which ("world"-)openness is organized in molecular genetics and which draws heavily upon individual scientists differs sharply from the recursive supra-epistemics of particle physics, whose main expedient I portrayed as the system's elaborate interaction with itself. Experience in this case appears to provide no more than an occasional touchstone which hurls the system back upon itself, and "success" may well depend on how well, and how intricately, the system interacts with itself. Experimental particle physics can indeed be likened to a cognitive system, in that it involves a complicated circuitry of recursive world-calculation, and in that its openness is organized within the walls of this calculation.

\section{Conclusion}

Let me stress that what $I$ have said should draw attention to the differences in procedure which we find in different fields' organization of openness and knowledge-grounding. These differences may point to the possibility of different relationships between these sciences' products. and the world, and to differences in the functioning of theories in different areas. The more interesting point, however, is what these differences suggest about "epistemics" (the grounding of knowledge) as a richly textured internal environment and culture. It can be shownthough I do not have the space to do so here-that in particle physics the relative loss of the empirical within its epistemic culture is matched by a loss of epistemic status of the individual, and that the elaborateness and recursiveness of its forms of reason corresponds to the elaborate- ness-and corporateness - of particle physics groups. Conversely, it can be shown that the interactional infra-epistemics of molecular genetics is supported by a special form of organization. As it appears, epistemic issues are inextricably intertwined with the organization of epistemic groups. And rationality must be seen more as a facet of the complex and tensely textured process which constitutes epistemic cultures. This paper was presented at the conference "Transitions in Recent Economics: Studies in
Alternative Research Programs," Capri, 15-19 October 1989. The research for this paper was supported by a grant from the Deutsche Forschungsgemeinschaft and was conducted with the help of the Center for Science Studies, University of Bielefeld.

\section{References}

Amann, K. 1990. Natürliche Expertise und künstliche Intelligenz - eine mikrosoziologische Untersuchung von Naturwissenschaftlern. Unpublished dissertation, University of Bielefeld.

Amann, K., and K. Knorr Cetina. 1988. The Fixation of (Visual) Evidence. Human Studies 11:133-69.

. 1989. Thinking through Talk: An Ethnographic Study of a Molecular Biology Laboratory. In Knowledge and Society: Studies in the Sociology of Science Past and Present, ed. R. A. Jones, L. Hargens, and A. Pickering, vol. 8. Greenwich, Conn.: Jai Press.

Barnes, B. 1974. Scientific Knowledge and Sociological Theory. London: Routledge \& Kegan Paul.

Bernstein, B. 1965. A Socio-Linguistic Approach to Social Learning. In Penguin Survey of the Social Sciences, ed. J. Gould. London: Penguin.

Bloor, D. 1976. Knowledge and Social Imagery. London: Routledge \& Kegan Paul.

Collins, H. M. 1985. Changing Order: Replication and Induction in Scientific Practice. London and Los Angeles: Sage.

de Marchi, N., ed. 1988. The Popperian Legacy in Economics. Cambridge: Cambridge University Press.

Giere, R. 1988. Explaining Science: A Cognitive Approach. Cambridge: Cambridge University Press.

Gooding, D., et al. 1989. The Uses of Experiment. Cambridge: Cambridge University Press.

Hacking, I. 1983. Representing and Intervening. Cambridge: Cambridge University Press.

Ingrao, B. 1989. The Hidden Epistemology of Rational Expectations and the Paradoxes of Rationality. Ricerche economiche 43.1-2:100-128.

Knorr Cetina, K. 1981. The Manufacture of Knowledge: An Essay on the Constructivist and Contextual Nature of Science. Oxford: Pergamon Press. . 1990. The Couch, the Cathedral and the Lab: On the Relationship between Experiment and Laboratory in Science. In Science as Practice and Culture, ed. A. Pickering. Chicago: University of Chicago Press.

Knorr Cetina, K., and K. Amann. 1988. Consensus Formation in the Laboratory. Paper prepared for the annual meeting of the American Sociological Association, Atlanta (August). 
1991. Image Dissection in Natural Scientific Inquiry. Science, Technology and Human Values 15.3:259-83.

Knorr Cetina, K., et al. 1988. Das naturwissenschaftliche Labor als Ort der "Verdichtung" von Gesellschaft. Zeitschrift für Soziologie 17.2:85-101.

1991. How Scientists Make Sense. In preparation

Lakatos, I. 1970. Falsification and the Methodology of Scientific Research Programmes. In Criticism and the Growth of Knowledge, edited by I. Lakatos and A. Musgrave. Cambridge: Cambridge University Press.

Latour, B., and S. Woolgar. 1979. Laboratory Life: The Social Construction of Scientific Facts. London and Beverly Hills: Sage.

Lynch, M. 1985. Art and Artifact in Laboratory Science: A Study of Shop Work and Shop Talk in a Research Laboratory. London: Routledge \& Kegan Paul. Mäki, U. 1989. On the Problems of Realism in Economics. Ricerche economiche 43.1-2:176-98.

Mannheim, K. 1936. Ideology and Utopia. New York: Harcourt, Brace \& World.

Maturana, H., and F. Varela. 1980. Autopoeisis and Cognition: The Realization of the Living. Boston.

Mead, G. H. 1967. Mind, Self and Society. Chicago: University of Chicago Press.

Merleau-Ponty, M. 1962. Phenomenology of Perception. London: Routledge \& Kegan Paul.

Merton, R. 1973. The Sociology of Science. Chicago: University of Chicago Press.

Mulkay, M. 1979. Science and the Sociology of Knowledge. London: George Allen \& Unwin.

Pickering, A. 1981. The Role of Interests in High Energy Physics: The Choice between Charm and Colour. In The Social Process of Scientific Investigation, edited by K. Knorr, R. Krohn, and R. Whitley. Social Sciences Yearbook, 4. Dordrecht: D. Reidel.

Ryle, G. 1971. Knowing How and Knowing That. In Collected Papers, vol. 2. London: Hutchinson.

Weintraub, E. R. 1985. General Equilibrium Analysis: Studies in Appraisal Cambridge: Cambridge University Press. 